gap $>\mathrm{g}:=$ SymmetricGroup $(4)$;

$\operatorname{Sym}\left(\left[\begin{array}{lll}1 & 4\end{array}\right]\right)$

15 : betti $(t$, Weights $\Rightarrow\{1$, gap $\}$ false

01234 gap> tblmod2:= CharacterTable( tbl, 2);

05 = total: 1413144 BrauerTable $(\operatorname{Sym}([1 \ldots 4]), 2$ )

1: . 2242 gap> tblmod2 = CharacterTable $(t b l, 2)$;

Journal of Software for

01234 gap> libtbl:= CharacterTable( "M" );

Algebra and Geometry

fail $\quad r i n g ~ r 1=32003,(x, y, z)$, ds;

gap> CharacterTable( "Symmetric", 4) ; int a,b,c,t=11,5,3,0;

BettiTally

CharactierTable ( "Sym(4)" )

gap> ComputedBrauerTables ( tbl );

$\begin{array}{lllll}0 & 1 & 2 & 3 & 4\end{array}$

[ poly $f=x^{\wedge} a+y^{\wedge} b+z^{\wedge}(3 * c)+x^{\wedge}(c+2) * y^{\wedge}(c-1)+x^{\wedge}$ $\mathrm{x}^{\sim}(\mathrm{c}-2) * \mathrm{y}^{\wedge} \mathrm{c} *\left(\mathrm{y}^{\sim} 2+\mathrm{t} * \mathrm{x}\right)^{\sim}-2$;

o7 = total: $14 \begin{array}{cccc}1 & 4 & 14 & 4\end{array}$

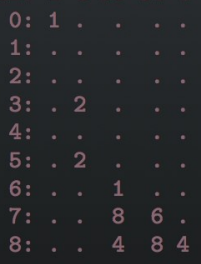

o7 : BettiTally

i8 : peek t1

$08=$ BettiTally $\{(0,\{0,0\}, 0) \Rightarrow 1\}$

$(1,\{2,2\}, 4) \Rightarrow 2$

$(1,\{3,3\}, 6) \Rightarrow 2$

(2, $\{3,7\}, 10) \Rightarrow 2$

(2, $\{4,4\}, 8) \Rightarrow 1$

(2, $\{4,5\}, 9) \Rightarrow 4$

$(2,\{5,4\}, 9) \Rightarrow 4$

(2, $\{7,3\}, 10) \Rightarrow 2$

(3, $\{4,7\}, 11) \Rightarrow 4$

$(3,47,44,11) \Rightarrow 4$ Algebra package in Macaulay2

$(4,\{5,7\}, 12) \Rightarrow 2$

$(4,\{7,5\}, 12) \Rightarrow 2$

DAVID EISENBUD 


\title{
The ReesAlgebra package in Macaulay2
}

\author{
DAVID EISENBUD
}

\begin{abstract}
This note introduces Rees algebras and some of their uses, with illustrations from version 2.2 of the Macaulay2 package ReesAlgebra.m2.
\end{abstract}

INTRODUCTION. A central construction in modern commutative algebra starts from an ideal $I$ in a commutative ring $R$, and produces the Rees algebra

$$
\mathcal{R}(I):=R \oplus I \oplus I^{2} \oplus I^{3} \oplus \cdots \cong R[I t] \subset R[t],
$$

where $R[t]$ denotes the polynomial algebra in one variable $t$ over $R$. For basics on Rees algebras, see [Vasconcelos 1994] and [Swanson and Huneke 2006], and for some other research, see [Eisenbud and Ulrich 2018; Kustin and Ulrich 1992; Ulrich 1994], and [Valabrega and Valla 1978].

From the point of view of algebraic geometry, the Rees algebra $\mathcal{R}(I)$ is a homogeneous coordinate ring for the graph of a rational map whose total space is the blowup of Spec $R$ along the scheme defined by $I$. (In fact, the "Rees algebra" is sometimes called the "blowup algebra".)

Rees algebras were first studied in the algebraic context by David Rees, in the now-famous paper [Rees 1958]. Actually, Rees mainly studied the ring $R\left[I t, t^{-1}\right]$, now also called the extended Rees algebra of $I$.

Mike Stillman and I wrote a Rees algebra script for Macaulay classic. It was augmented, and made into the [Macaulay2] package ReesAlgebra.m2 around 2002, to study a generalization of Rees algebras to modules described in [Eisenbud et al. 2003]. Subsequently Amelia Taylor, Sorin Popescu, the present author, and, at the Macaulay2 Workgroup in July 2017, Ilir Dema, Whitney Liske, and Zhangchi Chen contributed routines for computing many of the invariants of an ideal or module defined in terms of Rees algebras. These routines comprise the package's primary utility, since Rees algebras of modules other than ideals are comparatively little studied.

The author is grateful to the National Science Foundation for partial support.

MSC2010: primary 13A30, 13B22, 13D02; secondary 14C17, 14E15.

Keywords: Rees algebras, blowup, distinguished subvariety, special fiber.

ReesAlgebra.m2 version 2.2 
We first describe the construction and an example from [Eisenbud et al. 2003]. Then we list some of the functionality the package now has and illustrate it with a theorem of Morey and Ulrich. Finally we give examples of how Rees algebras appear in the Fulton-MacPherson intersection theory and in the resolution of singularities.

1. The Rees algebra of a module. There are several possible ways of extending the Rees algebra construction from ideals to modules. For simplicity we will henceforward only consider finitely generated modules over Noetherian rings. Huneke and Ulrich and I argued in [Eisenbud et al. 2003] that the most natural way to extend the definition is to think of $R[I t]$ as the image of the map of symmetric algebras $\operatorname{Sym}(\phi): \operatorname{Sym}_{R}(I) \rightarrow \operatorname{Sym}_{R}(R)=R[t]$, and to generalize it to the case of an arbitrary finitely generated module $M$ by setting

$$
\mathcal{R}(M)=\text { image } \operatorname{Sym}(\phi),
$$

where $\phi$ is a versal map from $M$ to a free module. Such a versal map may be computed as the composition of the diagonal embedding

$$
M \rightarrow \oplus_{i=1}^{m} M,
$$

with the map

$$
\oplus_{i=1}^{m} \phi_{i}: \oplus_{i=1}^{m} M \rightarrow R^{m}
$$

where $\phi_{1}, \ldots, \phi_{m}$ generate $\operatorname{Hom}_{R}(M, R)$.

Though this is not immediate, the Rees algebra of an ideal in a Noetherian ring, in this sense, is the same as the Rees algebra in the classical sense, and in most cases one can take any embedding of the module into a free module in the definition:

Theorem 1.1 [Eisenbud et al. 2003, Theorems 0.2 and 1.4]. Let $R$ be a Noetherian ring and let $M$ be a finitely generated $R$-module. Let $\phi: M \rightarrow G$ be a versal map of $M$ to a free module. Suppose that $\phi$ is an inclusion, and let $\psi: M \rightarrow G^{\prime}$ be any inclusion of $M$ into a free module $G^{\prime}$. If $R$ is torsion-free over $\mathbb{Z}$ or $R$ is unmixed and generically Gorenstein or $M$ is free locally at each associated prime of $R$, or $G^{\prime}=R$, then the image of $\operatorname{Sym}(\phi)$ and the image of $\operatorname{Sym}(\psi)$ are naturally isomorphic.

Nevertheless some examples do violate the conclusion of Theorem 1.1. Here is one from [Eisenbud et al. 2003] in characteristic 5 (any finite characteristic would work similarly).

i1 : $\mathrm{p}=5$;

i2: $R=Z Z / p[x, y, z] /\left(i d e a l\left(x^{\wedge} p, y^{\wedge} p\right)+(i d e a l(x, y, z))^{\wedge}(p+1)\right)$;

i3 : $M=$ module ideal $(z)$;

It is easy to check that $M \cong R^{1} /(x, y, z)^{p}$. We write $\iota: M \rightarrow R^{1}$ for the embedding as an ideal and $\psi$ for the embedding $M \rightarrow R^{2}$ sending $z$ to the vector $(x, y)$. 
i4 : iota $=\operatorname{map}\left(\mathrm{R}^{\wedge} 1, \mathrm{M}, \operatorname{matrix}\{\{z\}\}\right)$;

i5: $\operatorname{psi}=\operatorname{map}\left(\mathrm{R}^{\wedge} 2, \mathrm{M}, \operatorname{matrix}\{\{\mathrm{x}\},\{\mathrm{y}\}\}\right)$;

Finally, we choose a versal embedding $M \rightarrow R^{3}$. It sends $z$ to the vector $(x, y, z)$ : i6 $:$ phi $=$ versalEmbedding $(\mathrm{M})$;

We now compute the kernels of the three maps on symmetric algebras:

i7 : Iiota = symmetricKernel iota;

i8 : Ipsi = symmetricKernel psi;

i9 : Iphi = symmetricKernel phi;

and check that the ones corresponding to $\phi$ and $\iota$ are equal, whereas the ones corresponding to $\psi$ and $\phi$ are not — they differ in degree $p$.

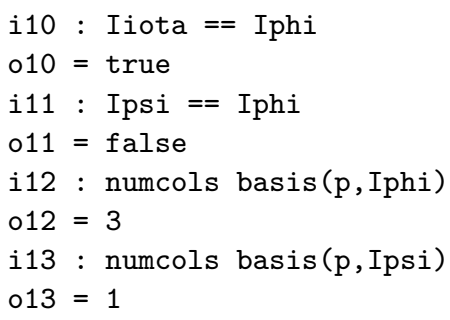

2. The ReEs Algebra AND its Relations. The central routine, reesIdeal (with synonym: reesAlgebraIdeal), computes an ideal defining the Rees algebra $\mathcal{R}(M)$ as a quotient of a polynomial ring over $R$ from a free presentation of $M$. From the Rees ideal we immediately get reesAlgebra $M$. In the case when $M$ is an ideal in $R$ we also compute the important associatedGradedRing $M=$ $\mathcal{R}(M) / M$ (and the more geometric sounding but identical normalCone $M$ ). If $I$ is a (homogeneous) ideal primary to the maximal ideal of a standard graded ring $R$ we compute the Hilbert-Samuel multiplicity of $I$ with the routine multiplicity.

We now describe the basic computation. Suppose that $M$ has a set of generators represented by a map from a free module,

$$
F \stackrel{\alpha}{\longrightarrow} M \rightarrow 0,
$$

and suppose $F=R^{n}$. The symmetric algebra of $F$ over $R$ is then a polynomial ring $\operatorname{Sym}_{R}(F)=R\left[t_{1}, \ldots, t_{n}\right]$ on $n$ new indeterminates $t_{1}, \ldots, t_{n}$. By the universal property of the symmetric algebra there is a canonical surjection $\operatorname{Sym}_{R}(F) \rightarrow$ $\operatorname{Sym}_{R}(M)$, so we may compute the Rees algebra of $M$ as a quotient of $\operatorname{Sym}_{R}(F)$. The expression

$$
I=\text { reesIdeal } M
$$

first uses versalEmbedding $M$ to compute a versal map from $M$ to a free module $\beta: M \rightarrow G$. The expression symmetricKernel $\alpha \circ \beta$ then constructs the map of 
symmetric algebras $\beta \circ \alpha: \operatorname{Sym}_{R}(F) \rightarrow \operatorname{Sym}_{R}(G)$ and uses the built-in Macaulay2 routine to compute the kernel

$$
I=\text { reesIdeal } M=\operatorname{ker} \operatorname{Sym}(\beta \circ \alpha): \operatorname{Sym}_{R}(F) \rightarrow \operatorname{Sym}_{R}(G) .
$$

There is a different way of computing the Rees algebra that is often much more efficient. It begins by constructing the symmetric algebra of $M$, and uses the observation that the construction of the Rees algebra commutes with localization. See [Eisenbud 1995, Appendix 2] for the necessary facts about symmetric algebras.

Suppose that $M$ has a free presentation,

$$
G \stackrel{\phi}{\longrightarrow} F \stackrel{\alpha}{\longrightarrow} M \rightarrow 0 .
$$

The right exactness of the symmetric algebra functor implies that the symmetric algebra of $M$ is the quotient of $\operatorname{Sym}_{R}(F)$ by an ideal $I_{0}$ that is generated by the entries of the matrix

$$
\left(t_{1} \cdots t_{n}\right) \circ \phi
$$

(where we have identified $\phi$ with $\operatorname{Sym}_{R}(F) \otimes_{R} \phi$ ). Thus $I_{0}$ is generated by polynomials that are linear in the variables $t_{i}$ (and because $M$ is the degree 1 part of $\mathcal{R}(M)$, these are the only linear forms in the $t_{i}$ in the Rees ideal).

If $f \in R$ is an element such that $M\left[f^{-1}\right]$ is free on generators $g_{1}, \ldots, g_{n}$, it follows that after inverting $f$, the Rees algebra of $M$ becomes a polynomial ring over $R\left[f^{-1}\right]$ on indeterminates corresponding to the $g_{i}$ :

$$
\mathcal{R}(M)\left[f^{-1}\right]=\operatorname{Sym}_{R}\left(M\left[f^{-1}\right]\right)=R\left[G_{1}, \ldots, G_{n}\right] .
$$

Now suppose in addition that $f$ is a non-zerodivisor in $R$. In the diagram

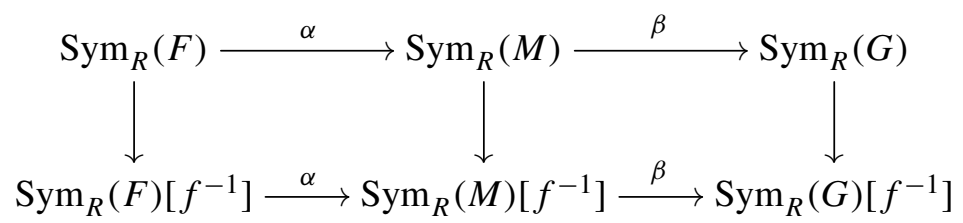

the two outer vertical maps are inclusions, and it follows that the Rees ideal, which is the kernel of the map $\mathcal{R}(F)=\operatorname{Sym}_{R}(F) \rightarrow \mathcal{R}(M)$, is equal to the intersection of $\mathcal{R}(F)$ with the kernel of

$$
\operatorname{Sym}_{R}(F)\left[f^{-1}\right] \stackrel{\beta}{\longrightarrow} \operatorname{Sym}_{R}(G)\left[f^{-1}\right] .
$$

This intersection may be computed as $I_{0}: f^{\infty}$. The command

$$
\text { reesIdeal }(I, f)
$$

computes the Rees ideal in this way.

More generally, we say that a module $N$ is of linear type if the Rees ideal of $M$ is equal to the ideal of the symmetric algebra of $M$; for example, any complete 
intersection ideal is of linear type, and the condition can be tested by the command

$$
\text { isLinearType } M \text {. }
$$

The procedure above really requires only that $f$ be a non-zerodivisor in $R$ and that $M\left[f^{-1}\right]$ be of linear type over $R\left[f^{-1}\right]$.

3. Reductions AND THE SPECIAL Fiber. A reduction $J$ of an ideal $I$ is a subideal $J \subset I$ over which $I$ is integrally dependent. In concrete terms this means that there is some integer $r$ such that $J I^{r}=I^{r+1}$, and the minimal $r$ with this property is called the reduction number. The property of being a reduction is tested by isReduction $I$, and reductionNumber $I$ computes the reduction number.

Now suppose that $\mathfrak{m}$ is a maximal ideal containing $I$. The special fiber ring is by definition $\mathcal{R}(I) / \mathfrak{m} \mathcal{R}(I)$. It is a standard graded algebra over the field $k:=R / \mathfrak{m}$, a quotient of $\operatorname{Sym}_{R}(F) / \mathfrak{m}=k\left[t_{1}, \ldots, t_{n}\right]$ where, as before, $F$ is a free module of rank $n$ with a surjection to $M$. The defining ideal of the special fiber ring, and the ring itself, are computed using specialFiberIdeal $I$ and specialFiberRing $I$.

The dimension of the special fiber ring is called the analytic spread of $I$, usually denoted

$$
\ell(I)=\text { analyticSpread } I .
$$

Northcott and Rees [1954] proved that if $k$ is infinite then there always exist reductions generated by $\ell(I)$ elements, and this is the minimum possible number; these are called minimal reductions. The smallest possible reduction number for $I$ with respect to a minimal reduction is by definition reductionNumber $I$. (This is always achieved by any ideal generated by $\ell(I)$ sufficiently general scalar linear combinations of the generators of $I$; but note that when $I$ is homogeneous but has generators of different degrees such linear combinations are sometimes necessarily inhomogeneous.)

An interesting special case occurs when $R$ is a graded ring over $k=R_{0}$ and the generators $g_{1}, \ldots, g_{n}$ of $I$ are all homogeneous of the same degree. In this case the special fiber ring is easily seen to be equal to the subring $k\left[g_{1}, \ldots, g_{n}\right]$ (usually not a polynomial ring) generated by the elements $g_{i}$.

For example, if $I$ is the ideal of $p \times p$ minors of a $p \times(p+q)$ matrix, then the special fiber ring is equal to the homogeneous coordinate ring $\mathbb{G}$ of the Grassmannian of $p$-planes in $p+q$ space. It follows that $\ell(I)=\operatorname{dim} \mathbb{G}=p q+1$, and the reduction number of $I$ is $(p-1)(q-1)$.

4. Finding elements of the Rees ideAl. Let $M$ be an $R$-module and let $\phi$ : $R^{s} \rightarrow R^{m}$ be its presentation matrix. We identify $\operatorname{Sym}_{R}\left(R^{m}\right)$ with the polynomial ring $R\left[t_{1}, \ldots, t_{m}\right]$. By the universality of the symmetric algebra construction, the 
symmetric algebra of $I$ has the form

$$
\operatorname{Sym}_{R}(I)=R\left[t_{1}, \ldots, t_{m}\right] /(T \phi),
$$

where we have written $T$ for the vector $\left(t_{1} \ldots t_{m}\right) \in R\left[t_{1}, \ldots, t_{m}\right]^{m}$, whose entries correspond to the generators of $I$, and written $(T \phi)$ for the ideal generated by the entries of the product

$$
\left(t_{1} \cdots t_{m}\right) \phi
$$

If $J:=\left(x_{1}, \ldots, x_{n}\right) \subset R$ is an ideal containing $I$, and we write

$$
X=\left(x_{1} \cdots x_{n}\right) \in R\left[t_{1}, \ldots, t_{m}\right]^{n},
$$

then there is a matrix $\psi$ defined over $R\left[t_{1}, \ldots, t_{m}\right]$, called the Jacobian dual of $\phi$ with respect to $X$, such that $T \phi=X \psi$. (The matrix $\psi$ is generally not unique; Macaulay2 computes it using Gröbner division with remainder.)

If $I, J$ each contain a non-zerodivisor then $J$ will have grade $\geq 1$ on the Rees algebra $\mathcal{R}(I)$. Since $(T \phi)$ is contained in the defining ideal of the Rees algebra, the vector $X$ is annihilated by the matrix $\psi$ when regarded over the Rees algebra, and the relation $X \psi \equiv 0$ in $\mathcal{R}(I)$ implies that the $m \times m$ minors of $\psi$ are in the Rees ideal of $I$.

In very favorable circumstances, one may even have the equality

$$
\text { reesIdeal } I==\text { ideal }(T \phi)+\operatorname{minors}(m, \psi) \text {. }
$$

We illustrate with a theorem of Morey and Ulrich. Recall that an ideal $I$ is said to satisfy the condition $G_{\ell}$ if the number of generators of the localized ideal $I_{P}$ is $\leq \operatorname{codim} P$ for every prime ideal $P$ of codimension $<\ell$; equivalently, if $I$ has presentation matrix $\phi$ as above,

$$
\operatorname{codim} I_{m-p}(\phi)>p
$$

for $1 \leq p<\ell$.

Theorem 4.1 [Morey and Ulrich 1996]. Let $R$ be a local Gorenstein ring with infinite residue field, let $I$ be a perfect ideal of grade 2 with $m$ generators, let $\phi$ be the presentation matrix of $I$, and let $\psi$ be the Jacobian dual matrix. Let $\ell=\ell(I)$ be the analytic spread. Suppose that I satisfies the condition $G_{\ell}$. The following conditions are equivalent:

(1) $\mathcal{R}(I)$ is Cohen-Macaulay and $I_{(m-\ell)}(\phi)=I_{1}(\phi)^{m-\ell}$.

(2) $r(I)<\ell$ and $I_{m+1-\ell} \phi=\left(I_{1} \phi\right)^{m+1-\ell}$.

(3) The ideal of $\mathcal{R}(I)$ is equal to the sum of the ideal of $\operatorname{Sym}(I)$ with the Jacobian dual minors, $I_{m} \psi$.

We can check all these conditions with functions in the package. We start with the presentation matrix $\phi$ of an $m=n+1$-generator perfect ideal such that the first 
row consists of the $n$ variables of the ring, and the rest of the rows are reasonably general (in this case random quadrics):

i2: setRandomSeed 0

i3 : $\mathrm{n}=3$;

i4: $\mathrm{kk}=\mathrm{ZZ} / 101$

i5 : $\mathrm{S}=\mathrm{kk}\left[\mathrm{a}_{-} 0 \ldots \mathrm{a}_{-}(\mathrm{n}-2)\right]$;

i6 : phi $=$ transpose $\operatorname{map}\left(\mathrm{S}^{\wedge}(\mathrm{n}-1), \mathrm{S}^{\wedge}\{-1,(\mathrm{n}-1):-2\}\right.$, $(i, j) \rightarrow$ if $j==0$ then $a_{-} i$ else $\left.\operatorname{random}(2, S)\right)$; 3

06 : Matrix $\mathrm{S}<---\mathrm{S}$

i7 : I $=\operatorname{minors}(\mathrm{n}-1, \mathrm{phi})$;

This is a perfect codimension 2 ideal, as we see from the Betti table:

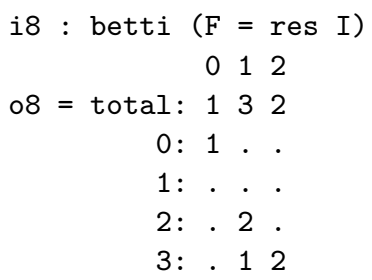

We compute the analytic spread $\ell$ and the reduction number $r$ :

i12 : ell = analyticSpread I

$012=2$

i13 : $r=$ reductionNumber $(I$, minimalReduction $I)$

$013=1$

Now we can check the condition $G_{\ell}$, first probabilistically:

i15 : whichGm I >= ell

$015=$ true

and now deterministically:

i17 : apply(toList(1. ell-1), $\mathrm{p}->\{\mathrm{p}+1, \operatorname{codim} \operatorname{minors}(\mathrm{n}-\mathrm{p}, \mathrm{ph} i)\})$

$017=\{\{2,2\}\}$

We now check the three equivalent conditions of the Morey-Ulrich theorem. Since $\ell=n-1$ in this case, the second parts of conditions (1) and (2) are vacuously satisfied, and since $r<\ell$ the conditions must all be satisfied. We first check that $\mathcal{R}(I)$ is Cohen-Macaulay:

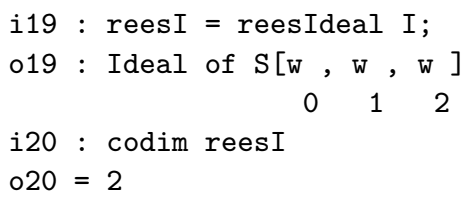




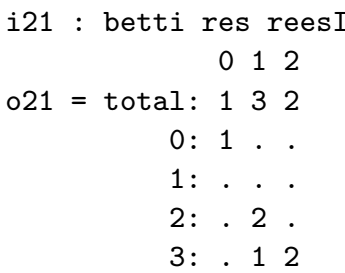

Finally, we wish to see that reesIdeal $I$ is generated by the ideal of the symmetric algebra together with the Jacobian dual:

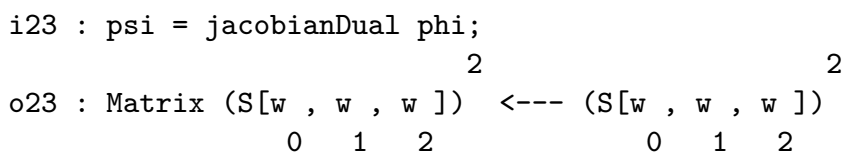

We now compute the ideal $J$ of the symmetric algebra; we do this by hand, since the command symmetricAlgebra $I$ would return the ideal over a different ring.

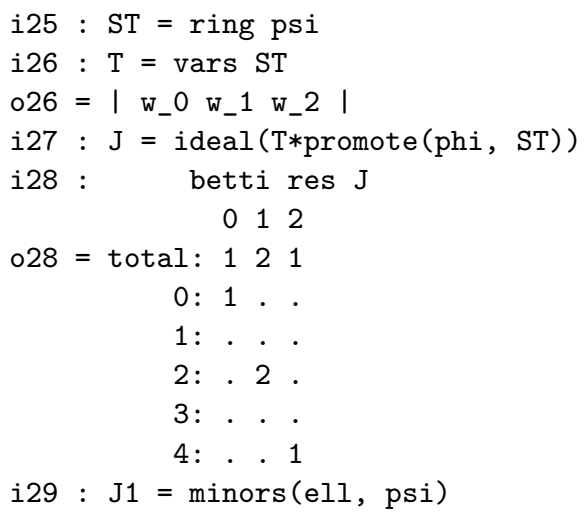

We compute the resolution of $G:=J+J 1$, to see that the resulting ideal is perfect, which also shows that it is the full ideal of the Rees algebra. We also check directly that it has the same resolution as the computed Rees ideal of $I$ :

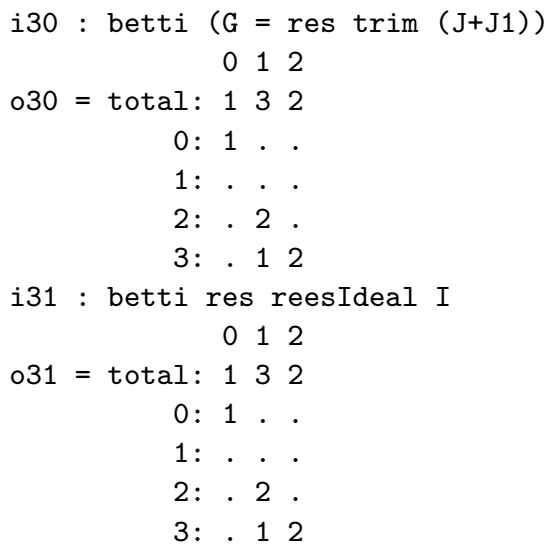


5. Distinguished SubVARIETIES. The key construction in the Fulton-MacPherson definition of the refined intersection product [Fulton 1998, Section 6.1] involves normal cones, and is easy to implement using the tools in this package. The simplest case is the intersection of two subvarieties $X, V \subset Y$. If $X$ and $V$ meet in the expected dimension, defined to be $\operatorname{dim} V-\operatorname{codim}_{Y} X$, and the ambient variety $Y$ is smooth, then one can assign multiplicities $m_{i}$ to the components $W_{i}$ of $X \cap V$, and the intersection product has the form $[X][V]=\sum m_{i}\left[W_{i}\right]$. The astonishing result of the Fulton-MacPherson theory is that if $X \subset Y$ is locally a complete intersection, then, no matter how singular $Y$ and no matter how strange the actual intersection $X \cap V$, the intersection product $X \cdot V$ can be given a meaning as a rational equivalence class of cycles of the expected dimension on $X$, or even on certain distinguished subvarieties $Z_{i}$ of $X \cap V$. This class comes with a canonical decomposition $\sum_{i} m_{i} \alpha_{i}$, where the $m_{i}$ are positive integers, and $\alpha_{i}$ is a cycle of the expected dimension (possibly 0 ) on $Z_{i} \subset X \cap V$ (the same $Z_{i}$ can appear several times, with different multiplicities and cycles).

In the general case, the subvariety $V$ is replaced by a morphism $f: V \rightarrow Y$ from a variety $V$, and this is the key to the functoriality of the intersection product. The routines in this package work in the general setting, but for simplicity we will stick with the basic case in this description.

We now describe the distinguished subvarieties and their multiplicities. This part of the construction sheafifies, so (as in the package) we work in the affine case. We do not require any hypothesis on $X, Y$ or $V$.

Let $S$ be a ring (for example, the coordinate ring of $Y$ ) and let $I \subset S$ be an ideal (for example, the ideal of $X$ ). Write

$$
T:=\operatorname{gr}_{I} S=S / I \oplus I / I^{2} \oplus \cdots
$$

for the associated graded ring of $I$, and let $\pi$ be the inclusion of $S / I$ into $T$ as the degree 0 part.

Let $f: S \rightarrow R$ be a ring homomorphism (for example, representing the projection $S \rightarrow S /(I(V)))$. Let $K \subset T$ be the kernel of the induced map $\operatorname{gr}_{I} S \rightarrow \operatorname{gr}_{f(I) R} R$.

Let $P_{1}, \ldots, P_{m}$ be the minimal primes over $K$ in $\operatorname{gr}_{I} R$. We define $p_{i}$ to be the degree 0 part of $P_{i}$; that is, $p_{i}:=P_{i} \cap S / I$. These are the distinguished prime ideals of $S / I$, and they clearly contain the kernel of $\bar{f}: S / I \rightarrow R / f(I) R$, so in the case where $R=S / J$ they contain $I+J$. Thus, in this case, they represent subvarieties of $X \cap V$.

Let $m_{i}$ be the multiplicity with which $P_{i}$ appears in the primary decomposition of $K$ - that is,

$$
m_{i}:=\text { length }_{\kappa\left(P_{i}\right)} P_{i P_{i}} / K_{P_{i}},
$$

where $\kappa\left(P_{i}\right)=T_{P_{i}} / P_{i_{P_{i}}}$ is the residue field at $P_{i}$. Returning to geometric language, and the case where $X \subset Y$ is locally a complete intersection in a quasiprojective 
variety, the cycle class $\alpha_{i}$ in the Chow group of the variety $Z_{i}$ corresponding to $p_{i}$ is defined as the Gysin image of the class of the subvariety corresponding to $P_{i}$ in the projectivized normal bundle of $X$ in $Y-$ a construction not included in this package.

Here are some simple examples in which distinguished is used to compute the distinguished varieties of intersections in $\mathbb{A}^{n}$, via the function intersect InP. First, the familiar multiplicity 2 intersection of a conic with a tangent line.

i2 : $\mathrm{kk}=\mathrm{ZZ} / 101$;

i3 : $\mathrm{P}=\mathrm{kk}[\mathrm{x}, \mathrm{y}]$;

i4: I = ideal"x2-y"; J=ideal y;

$i 6$ : $i n t e r \operatorname{sectInP}(I, J)$

$06=\{\{2$, ideal $(\mathrm{y}, \mathrm{x})\}\}$

Slightly more interesting, the following shows what happens when the intersections aren't rational:

i7: $I$ = ideal" $x 4+y 3+1 "$;

i8 : intersectInP $(\mathrm{I}, \mathrm{J})$

$08=\left\{\left\{1\right.\right.$, ideal $\left.\left(y, x^{2}+10\right)\right\},\left\{1\right.$, ideal $\left.\left.\left(y, x^{2}-10\right)\right\}\right\}$

The real interest in the construction is in the case of improper intersections. Here are some typical results:

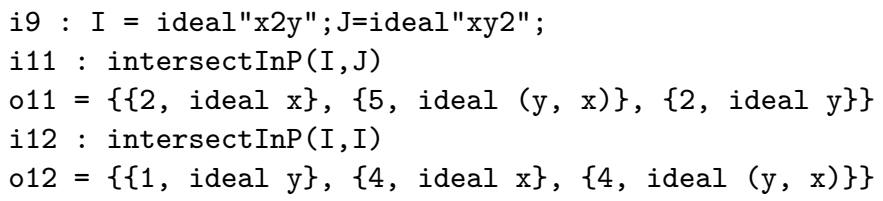

6. ReEs Algebras And Desingularization. We conclude with an example illustrating a general result about projective birational maps of varieties. Recall that a map $B \rightarrow X$ of varieties is projective if it is the composition of a closed embedding $B \subset X \times \mathbb{P}^{n}$ with the projection to $X$. It is birational if it is generically an isomorphism. The inclusion of a ring into the Rees algebra of an ideal corresponds to a map from Proj of the Rees algebra to Spec of the ring, called a blowup, that is such a proper birational transformation, and in fact every proper birational transformation to an affine variety (or more generally to any scheme, if one works with sheaves of ideals) can be realized in this way.

The theorem of embedded resolution of singularities (proven by Hironaka in characteristic 0 and conjectured in general) says that, given any subvariety $X$ of a smooth variety $Y$, there is a finite sequence of blowups

$$
B_{n} \rightarrow \cdots \rightarrow B_{2} \rightarrow B_{1} \rightarrow Y
$$

of smooth subvarieties that lie over the singular set of $X$, and a component of the preimage of $X$ in $B_{n}$ that is smooth and maps birationally to $X$. In the case of 
plane curves, this can be done with a sequence of blowups of closed points. But in fact any sequence of blowups of a quasiprojective variety can be replaced with a single blowup [Hartshorne 1977, Theorem II.7.17] of a more complicated ideal. We illustrate this with the desingularization of a tacnode (the union of two smooth curves that meet with a simple tangency).

Example 6.1. Blowing-up $\left(x^{2}, y\right)$ in $k[x, y]$ desingularizes the tacnode $x^{2}-y^{4}$ in a single step.

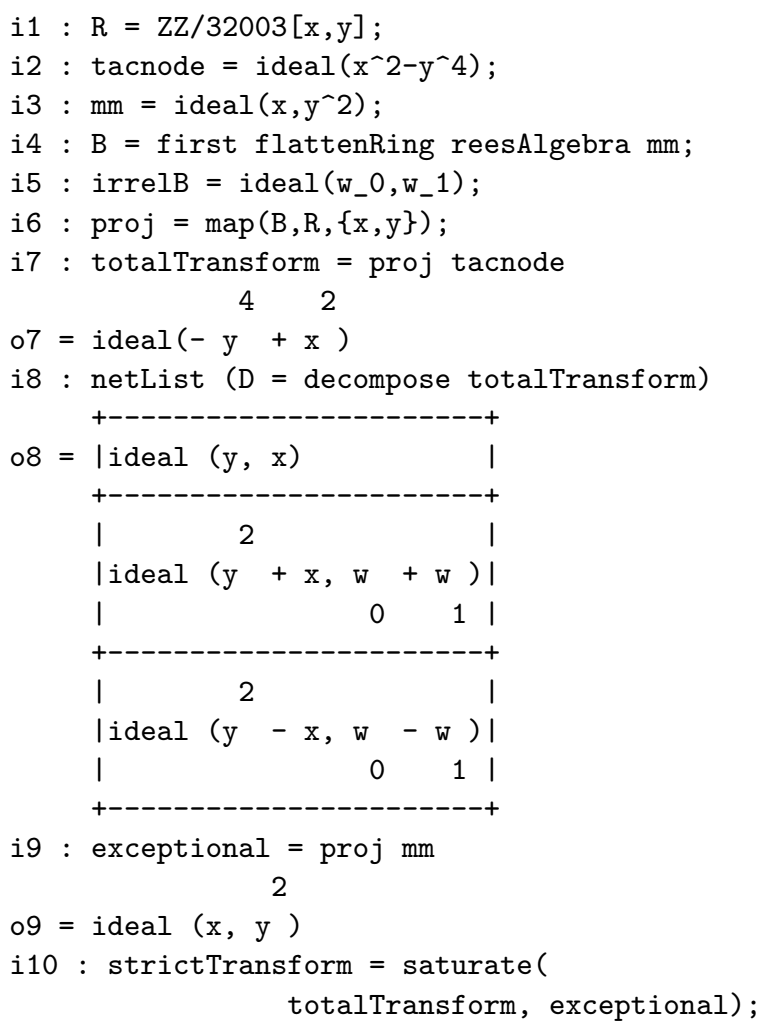


The last line asserts that the singular locus of the strict transform is empty; that is, the scheme defined by strictTransform is smooth (in this case it is the union of two disjoint smooth curves).

SUPPLEMENT. The online supplement contains version 2.2 of ReesAlgebra.m2.

\section{REFERENCES.}

[Eisenbud 1995] D. Eisenbud, Commutative algebra with a view toward algebraic geometry, Graduate Texts in Mathematics 150, Springer, 1995. MR Zbl

[Eisenbud and Ulrich 2018] D. Eisenbud and B. Ulrich, "Duality and socle generators for residual intersections", Journal für die reine and angewandte Mathematik (online publication July 2018).

[Eisenbud et al. 2003] D. Eisenbud, C. Huneke, and B. Ulrich, "What is the Rees algebra of a module?", Proc. Amer. Math. Soc. 131:3 (2003), 701-708. MR Zbl

[Fulton 1998] W. Fulton, Intersection theory, 2nd ed., Ergebnisse der Mathematik (3) 2, Springer, 1998. MR Zbl

[Hartshorne 1977] R. Hartshorne, Algebraic geometry, Graduate Texts in Mathematics 52, Springer, 1977. MR Zbl

[Kustin and Ulrich 1992] A. R. Kustin and B. Ulrich, "A family of complexes associated to an almost alternating map, with applications to residual intersections", Mem. Amer. Math. Soc. 95:461 (1992), iv+94. MR

[Macaulay2] D. R. Grayson and M. E. Stillman, "Macaulay2: a software system for research in algebraic geometry", available at http://www.math.uiuc.edu/Macaulay2.

[Morey and Ulrich 1996] S. Morey and B. Ulrich, "Rees algebras of ideals with low codimension", Proc. Amer. Math. Soc. 124:12 (1996), 3653-3661. MR Zbl

[Northcott and Rees 1954] D. G. Northcott and D. Rees, "Reductions of ideals in local rings", Proc. Cambridge Philos. Soc. 50 (1954), 145-158. MR Zbl

[Rees 1958] D. Rees, “On a problem of Zariski”, Illinois J. Math. 2 (1958), 145-149. MR Zbl

[Swanson and Huneke 2006] C. Huneke and I. Swanson, Integral closure of ideals, rings, and modules, London Mathematical Society Lecture Note Series 336, Cambridge University Press, 2006. MR Zbl

[Ulrich 1994] B. Ulrich, "Artin-Nagata properties and reductions of ideals", pp. 373-400 in Commutative algebra: syzygies, multiplicities, and birational algebra (South Hadley, MA, 1992), edited by W. J. Heinzer et al., Contemp. Math. 159, Amer. Math. Soc., Providence, RI, 1994. MR Zbl

[Valabrega and Valla 1978] P. Valabrega and G. Valla, "Form rings and regular sequences", Nagoya Math. J. 72 (1978), 93-101. MR

[Vasconcelos 1994] W. V. Vasconcelos, Arithmetic of blowup algebras, London Mathematical Society Lecture Note Series 195, Cambridge University Press, 1994. MR Zbl

ReCEIVED: 19 Aug 2017 Revised: 4 Feb 2018 ACCEPTED: 21 May 2018

\section{DAVID EISENBUD:}

de@msri.org

Mathematical Sciences Research Institute, Berkeley, CA, United States 

HeLP: a GAP package for torsion units in integral group rings

Andreas Bächle and Leo Margolis

A software package to compute automorphisms of graded algebras

Simon Keicher

A package for computations with classical resultants

Giovanni Staglianò

The Space Curves package in Macaulay2

Mengyuan Zhang

The ReesAlgebra package in Macaulay2

David Eisenbud

A Macaulay2 package for computations with rational maps

Giovanni Staglianò

ExteriorIdeals: a package for computing monomial ideals in an exterior algebra

Luca Amata and Marilena Crupi

Software for computing conformal block divisors on $\bar{M}_{0, n}$

David Swinarski

Divisor Package for Macaulay2

Karl Schwede and Zhaoning Yang 